\title{
SARS-CoV2 vaccines: Slow is fast
}

\author{
Douglas R. Green
}

Deputy Editor, Science Advances; Department of Immunology, St. Jude Children's Research Hospital, Memphis, TN 38105 USA. Email: Douglas.Green@STJUDE.0RG

Catastrophic numbers of infections, morbidities, and mortalities during the COVID-19 pandemic, together with the disastrous impact on world economies have mandated a historically unparalleled effort to produce an effective vaccine. There are currently 95 vaccines in development against SARS-CoV2, the causative virus of COVID-19 (1), with several in, or entering clinical trials. Most of these are expected to clear Phase I and two experimental vaccines have moved into Phase II trials.

An effective vaccine is the best route to a return to "normalcy." The only alternatives are widespread, systematic testing of the population with rigorous tracing of contacts, or readily available therapeutics to effectively treat infection and disease. Until the pandemic is resolved, social distancing, isolation, and personal protective equipment remain the best defense against infection.

The need to develop a vaccine is urgent, but most estimates conclude that even if trials are successful, an effective vaccine will not be widely available for 12 to 18 months. Much of the required time is related to the pace of proper clinical trials. Studies are usually first needed in experimental animals. However, non-human primates rarely display severe symptoms, and critical disease is also not observed in other species, including genetically engineered mice.

Clinical trials proceed in three phases. In Phase I and II the vaccine is tested on a small cohort of individuals to determine if the vaccine is safe in the short term and elicits immune responses. A Phase III trial follows, which tests the vaccine in a large cohort for actual efficacy against infection and potential adverse effects. Phase III trials are necessarily time-consuming and require that infection among the control group is sufficiently high to draw a conclusion.

Many advocate "fast-tracking" these trials, and some wish to rely soley on evidence of induction of neutralizing responses. However, this could be catastrophic. In 1966, a large trial of a vaccine for Respiratory Syncytial Virus (RSV) found that the immunized cohort actually faired significantly worse upon infection. There is some reason to worry that the same may occur with some SARS-CoV2 vaccines.

Any immune response is of two types, adaptive or innate. The innate response is not specific to an infection and is usually considered a first line of defense. However, there is evidence that innate responses can be "trained" to respond more rapidly to infection. Vaccination with an attenuated tuberculosis bacterium (BCG), can protect children against respiratory infections. At least one clinical trial has been initiated to investigate the efficacy of BCG for protection of health care workers exposed to SARS-CoV2 (2).

Adaptive immunity is elicited by most vaccines and is mediated by lymphocytes. B lymphocytes produce antibodies, some of which neutralize the virus. This response requires the participation of $\mathrm{CD} 4{ }^{+} \mathrm{T}$ cells, which are required for optimal B cell responses, and for antibody affinity maturation, the production of strongly binding antibodies over time. With the failed RSV vaccine, a lack of antibody affinity maturation caused the negative effect. Adaptive immunity also elicits $\mathrm{CD}^{+} \mathrm{T}$ cells, capable of specifically killing virally infected cells. A vaccine that elicits only one of these is often effective in "shifting the balance" of the infection so that disease, if it occurs, is mild. Studies of T cell responses to SARS-CoV (the corona virus responsible for SARS) found that positive clinical outcome correlated with the numbers of virus-specific $\mathrm{CD} 4{ }^{+} \mathrm{T}$ cells.

The SARS-CoV2 genome encodes four structural proteins: the spike protein ( $\mathrm{S}$, which is responsible for recognition and entry into cells via binding to its receptor, Angiotensin I Converting Enzyme, ACE2); the membrane glycoprotein (M); the nucleocapsid protein $(\mathrm{N})$; and the envelope protein (E). It also encodes 16 non-structural proteins (NSPs) and 9 accessory proteins. Antibodies to the S protein have the potential to prevent viral entry, while antibodies to other structural proteins can also potentially promote viral destruction. In principle, $\mathrm{CD}^{+} \mathrm{T}$ cells specific for any of the viral proteins would kill virally infected cells. A study of SARS-CoV in hamsters concluded that only the S protein elicited effective immunity to infection (3). Most of the vaccines in development that involve a single protein focus on the $\mathrm{S}$ protein.

There are potential reasons why an immune response to a vaccine can predispose an individual to a worse outcome upon infection. One is the phenomenon of antibody-dependent enhancement (ADE). In this effect, antibodies that bind to the virus also bind to antibody receptors on cells, facilitating uptake and infection of the cell bearing the receptors. $\mathrm{ADE}$ has been observed for vaccines against Dengue, Ebola, and HIV (4). As recently as 2017, a large-scale efficacy trial of a Dengue vaccine resulted in ADE in vaccinated children (5). Troublingly, ADE has also been seen with vaccines for a feline coronavirus $(6,7)$. There is also evidence for ADE in SARSCoV. Studies have shown that rodent and human antibodies to the $\mathrm{S}$ protein can enhance infection in vitro (8-11). 
However, several small preclinical studies of a SARS-CoV vaccine in rhesus monkeys failed to observe evidence of $\mathrm{ADE}$.

One SARS-CoV2 vaccine, employing inactivated virus, was tested in several large cohorts of rhesus monkeys, with substantial efficacy and no evidence of ADE (12). While this is clearly encouraging, the need to ensure that any vaccine is, indeed, safe is of vital importance.

While there is a need for caution, the need for accelerating testing is clear, and many discussions are occurring on how to ethically perform experimental infection studies in humans. Such studies provide enormous progress in vaccine research, but of course the extreme risks must be weighed against potential benefits. The same applies to the ultimate use of a vaccine that shows adverse effects in clinical testing.

Referring to experimental research, the eminent cancer biologist, Charles Sherr once told me, "Fast is slow, and slow is fast." This is a maxim that must be applied to vaccine development for COVID-19.

\section{REFERENCES AND NOTES}

1. https://www.bioworld.com/COVID19products\#vac1

2. https:/clinicaltrials.gov/ct2/show/NCT04348370

3. U. J. Buchholz, A. Bukreyev, L. Yang, E. W. Lamirande, B. R. Murphy, K. Subbarao, P. L. Collins, Contributions of the structural proteins of severe acute respiratory syndrome coronavirus to protective immunity. Proc. Natl. Acad. Sci. U.S.A. 101, 9804-9809 (2004). doi:10.1073/pnas.0403492101 Medline

4. A. Takada, Y. Kawaoka, Antibody-dependent enhancement of viral infection: Molecular mechanisms and in vivo implications. Rev. Med. Virol. 13, 387-398 (2003). doi:10.1002/rmv.405 Medline

5. S. B. Halstead, Dengvaxia sensitizes seronegatives to vaccine enhanced disease regardless of age. Vaccine 35, 6355-6358 (2017). doi:10.1016/i.vaccine.2017.09.089 Medline

6. T. Hohdatsu, M. Nakamura, Y. Ishizuka, H. Yamada, H. Koyama, A study on the mechanism of antibody-dependent enhancement of feline infectious peritonitis virus infection in feline macrophages by monoclonal antibodies. Arch. Virol. 120, 207-217 (1991). doi:10.1007/BF01310476 Medline

7. T. Takano, S. Yamada, T. Doki, T. Hohdatsu, Pathogenesis of oral type I feline infectious peritonitis virus (FIPV) infection: Antibody-dependent enhancement infection of cats with type I FIPV via the oral route. J. Vet. Med. Sci. 81, 911-915 (2019). doi:10.1292/jvms.18-0702 Medline

8. M. Jaume, M. S. Yip, C. Y. Cheung, H. L. Leung, P. H. Li, F. Kien, I. Dutry, B. Callendret, N. Escriou, R. Altmeyer, B. Nal, M. Daëron, R. Bruzzone, J. S. Peiris, Anti-severe acute respiratory syndrome coronavirus spike antibodies trigger infection of human immune cells via a $\mathrm{pH}$ - and cysteine protease-independent $\mathrm{Fc} \gamma \mathrm{R}$ pathway. J. Virol. 85, 10582-10597 (2011). doi:10.1128/JVI.00671-11 Medline

9. Y. W. Kam, F. Kien, A. Roberts, Y. C. Cheung, E. W. Lamirande, L. Vogel, S. L. Chu, J. Tse, J. Guarner, S. R. Zaki, K. Subbarao, M. Peiris, B. Nal, R. Altmeyer, Antibodies against trimeric $S$ glycoprotein protect hamsters against SARS-CoV challenge despite their capacity to mediate FcgammaRII-dependent entry into B cells in vitro. Vaccine 25, 729-740 (2007). doi:10.1016/i.vaccine.2006.08.011 Medline

10. L. Liu, Q. Wei, Q. Lin, J. Fang, H. Wang, H. Kwok, H. Tang, K. Nishiura, J. Peng, Z. Tan, T. Wu, K. W. Cheung, K. H. Chan, X. Alvarez, C. Qin, A. Lackner, S. Perlman, K. Y. Yuen, Z. Chen, Anti-spike IgG causes severe acute lung injury by skewing macrophage responses during acute SARS-CoV infection. JCI Insight 4, 4 (2019). doi:10.1172/jci.insight.123158 Medline

11. S. F. Wang, S. P. Tseng, C. H. Yen, J. Y. Yang, C. H. Tsao, C. W. Shen, K. H. Chen, F. T. Liu, W. T. Liu, Y. M. Chen, J. C. Huang, Antibody-dependent SARS coronavirus infection is mediated by antibodies against spike proteins. Biochem. Biophys. Res. Commun. 451, 208-214 (2014). doi:10.1016/j.bbrc.2014.07.090 Medline

12. https://doi.org/10.1101/2020.04.17.046375
Published First Release 22 May 2020

10.1126/sciadv.abc7428 